\title{
Pneumomediastinum complicating adult-onset measles
}

\author{
Authors: Azara Janmohamed, ${ }^{\mathrm{A}}$ Aula Abbara, ${ }^{\mathrm{B}}$ Rohma Ghani, ${ }^{\mathrm{C}}$ Anne Kinderlerer, ${ }^{\mathrm{D}}$ Thava Sritharan ${ }^{\mathrm{E}}$ and \\ James Hatcher ${ }^{\mathrm{F}}$
}

\begin{abstract}
Adult-onset measles is rare in the UK, particularly in patients with a complete vaccination history. We present a case of a UK-born patient who received all childhood vaccinations, had no history of recent travel or unwell contacts who was diagnosed with measles complicated by pneumomediastinum. This case highlights the need to consider measles in any patient presenting with a constellation of a macular rash, fever and conjunctivitis, regardless of vaccination status. The nature of the rash can provide an important clue to the diagnosis. Liaison with infection specialists facilitates early diagnosis, allowing for appropriate initial investigations, improving clinical management and early infection control precautions being instituted.
\end{abstract}

KEYWORDS: Measles, pneumomediastinum, rash, conjunctivitis, vaccination

\section{Case presentation}

A 30-year-old man presented with a 5-day history of fever, a sore throat, a severe cough and a rash. The rash was macular, blanching and had a centrifugal spread, starting on his forehead and progressing to his chest, trunk and limbs. He reported reddening of his eyes and loose stools. He was short of breath and described being unable to take deep breaths.

He had no previous medical problems, no travel history, no unwell contacts and reported a history of having received all childhood vaccinations (this was confirmed by his mother who is a nurse).

On examination, he was febrile at $38.9^{\circ} \mathrm{C}$, with a blood pressure of $172 / 87 \mathrm{mmHg}$ and a heart rate of 122 beats per minute.

Authors: ${ }^{\text {A }}$ specialist registrar, clinical pharmacology and therapeutics and general medicine, Imperial College Healthcare NHS Trust, London, UK; ${ }^{B}$ consultant, infectious diseases and general medicine, Imperial College Healthcare NHS Trust, London, UK; ' specialist registrar, infectious diseases and microbiology, Imperial College Healthcare NHS Trust, London, UK; D ${ }^{\text {consultant, }}$ rheumatology and acute medicine, Imperial College Healthcare NHS Trust, London, UK; ${ }^{\mathrm{E}}$ consultant, geriatrics and general medicine, Imperial College Healthcare NHS Trust, London, UK; ${ }^{F}$ consultant, infectious diseases and microbiology, Imperial College Healthcare NHS Trust, London, UK

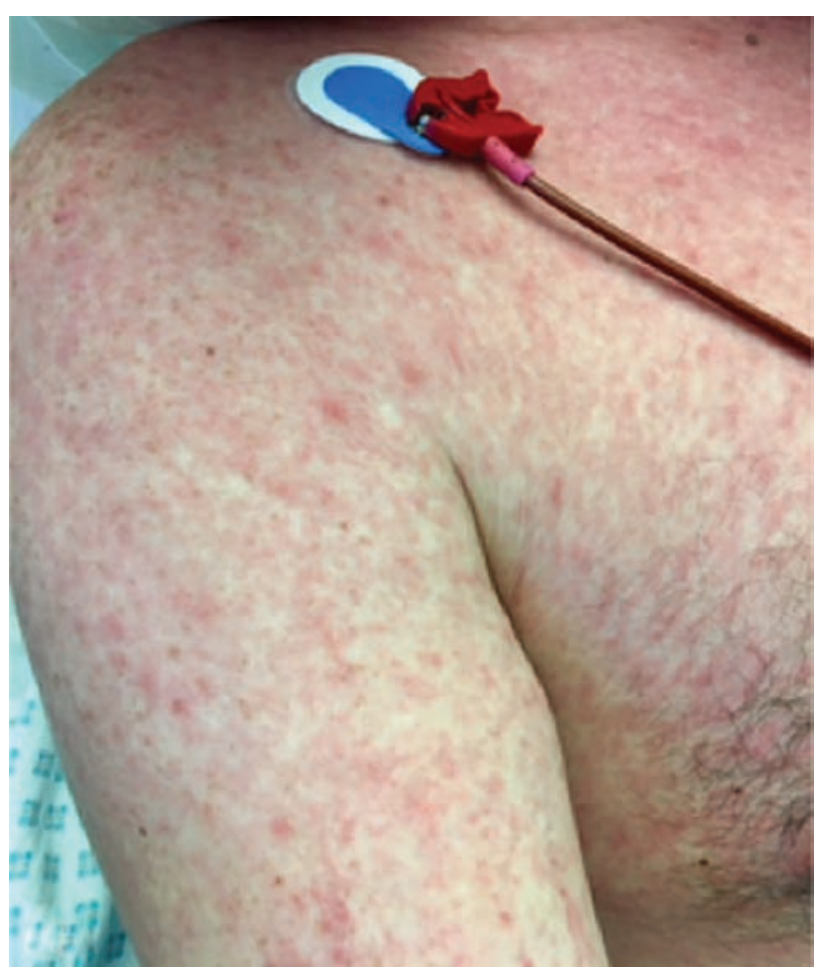

Fig 1. Blanching macular rash on trunk.

Peripheral saturations were $94 \%$ on room air. An arterial blood gas on air showed $\mathrm{pH} 7.49, \mathrm{pCO}_{2} 4.8, \mathrm{pO}_{2}$ 8.1, and lactate 0.8 . He had bilateral conjunctivitis and a widespread, blanching macular rash involving the face, trunk and extremities, as well as the palms and soles (Fig 1).

His blood tests revealed thrombocytopenia with platelets at $90 \times 10^{9} / \mathrm{L}$ (normal range $133-370 \times 10^{9} / \mathrm{L}$ ), lymphopenia at $0.9 \times$ $10^{9} / \mathrm{L}\left(1.1-3.6 \times 10^{9} / \mathrm{L}\right)$, haemoglobin $(\mathrm{Hb}) 176 \mathrm{~g} / \mathrm{L}(114-150 \mathrm{~g} / \mathrm{L})$, white cell count (WCC) $6.4 \times 10^{9} / \mathrm{L}\left(4.2-11.2 \times 10^{9} / \mathrm{L}\right)$, sodium $(\mathrm{Na})$ $131 \mathrm{mmol} / \mathrm{L}$ (133-146 mmol/L), Potassium (K) $3.6 \mathrm{mmol} / \mathrm{L} \mathrm{(3.5-5.3}$ $\mathrm{mmol} / \mathrm{L})$, creatinine $(\mathrm{Cr}) 105 \mu \mathrm{mol} / \mathrm{L}(55-110 \mu \mathrm{mol} / \mathrm{L})$, urea (Ur) $5.5 \mathrm{mmol} / \mathrm{L}$ (2.5-7.8 mmol/L), and C-reactive peptide (CRP) 184.7 $\mathrm{mg} / \mathrm{L}(0-5 \mathrm{mg} / \mathrm{L})$. A HIV test was negative.

The patient's admission chest $X$-ray showed a pneumomediastinum and pneumopericardium (Fig 2). 


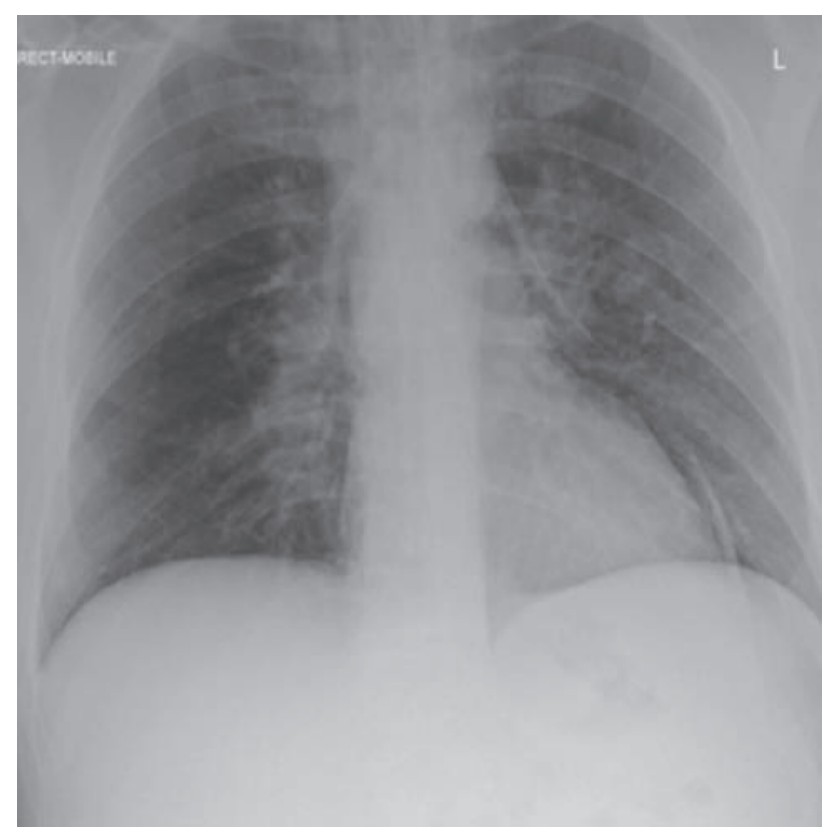

Fig 2. Chest X-ray showing pneumomediastinum and pneumopericardium

\section{Differential diagnosis}

The initial diagnosis was unclear. The patient was reviewed by numerous medical teams with specialties in acute medicine, rheumatology, dermatology, and ear, nose and throat (ENT). A wide differential was established including bacterial sepsis, acute rheumatological presentation (granulomatosis with polyangiitis) or a non-specific viral illness. On day two of his admission, the patient was reviewed by infectious diseases specialists who suggested the possibility of measles.

\section{Initial management}

The patient received supportive management with intravenous fluids, oxygen and intravenous antibiotics (ceftriaxone $2 \mathrm{gm}$ once daily and metronidazole $500 \mathrm{mg}$ three times daily).

A subsequent computed tomography (CT) chest confirmed that the pneumomediastinum extended to the base of the skull; oral gastrograffin contrast showed no evidence of oesophageal rupture. The patient was discussed with cardiothoracic surgeons who recommended conservative management for the pneumomediastinum.

\section{Case progression and outcome}

Diagnostic tests revealed positive measles IgM serology and positive real-time polymerase chain reaction (RT-PCR) for the measles genome in the patient's serum sample and throat swab (Genotype B3).

He was managed supportively with high-flow oxygen, fluid management and antimicrobials. Five days after his admission, he improved and was discharged home. Follow-up chest X-ray showed resolution of the pneumomediastinum.

\section{Discussion}

Adult-onset measles is rare in the UK, particularly in patients with a complete vaccination history. In September 2017, the World Health Organization (WHO) confirmed that the UK had achieved measles elimination in 2016, with no endemic measles transmission. However, imported cases, particularly cases that are imported from Europe, continue to be seen with some leading to onward transmission in the UK. Around half of cases are seen among adults, with $61 \%$ requiring hospitalisation in the third quarter of 2017 .

Measles is noted for its high rate of transmission. This can be up to $90 \%$ in susceptible domestic contacts. Vigilance should be exercised when a patient presents with a high fever, conjunctivitis, cough and coryza and then goes on (2-4 days later) to develop the characteristic rash that spreads from the face and trunk to extremities. Early consideration of measles as a diagnosis is important to ensure appropriate infection prevention and control measures.

Serious complications of measles can occur rarely. These include pneumonia and encephalitis, predominantly affecting children under 5 years and adults over 30 years. ${ }^{2}$

Here, we present the case of a UK-born patient who received all childhood vaccinations and had no history of recent travel or unwell contacts, who was diagnosed with measles complicated by pneumomediastinum.

Outbreaks of measles in vaccinated populations rarely occur and are termed vaccine failures. About 3 out of 100 people who get two doses of the MMR (measles, mumps and rubella) vaccine will get measles if exposed to the virus. However, they are more likely to have a milder illness and are less likely to spread the disease to other people. ${ }^{3}$ Our case represents a vaccine failure but was not associated with a subsequent outbreak.

Pneumomediastinum (also known as mediastinal emphysema) with measles has been reported in the literature, particularly in children, but reports involving adults are rare. ${ }^{4}$ It probably results from an increase in alveolar pressure leading to alveolar rupture, which allows air to extend into mediastinal borders. In this patient, the pneumomediastinum was managed conservatively with highflow oxygen and supportive care.

The management of measles is essentially supportive and involves fluids, antipyretics and the treatment of secondary bacterial infections such as pneumonia and otitis, which occur secondary to the profound immunosuppression that follows measles infection. ${ }^{5}$ Timely tracing of contacts should be carried out and post-exposure prophylaxis should be considered for unvaccinated contacts.

\section{Key learning points}

> Consider measles in any patient presenting with a constellation of a macular rash, fever and conjunctivitis, regardless of vaccination status. The nature of the rash (centrifugal as compared to centripetal) can provide an important clue to the diagnosis

> Adult onset measles is rare but can occur in the UK in patients with a good vaccination history

> Liaison with infection specialists facilitates early diagnosis, allowing for appropriate initial investigations, improving clinical management and the institution of early infection control precautions 
> Rare complications of measles include pneumomediastinum secondary to alveolar rupture and air-leak syndrome

$>$ The management of measles is supportive and involves fluids, antipyretics and the treatment of secondary bacterial infections

> Post-exposure prophylaxis should be considered in unvaccinated contacts with timely tracing of contacts.

\section{Consent to publish}

Written consent was obtained to publish the clinical details and images in this article.

\section{References}

1 Public Health England. Laboratory confirmed cases of measles, mumps and rubella, England: July to September 2017. www. gov.uk/government/uploads/system/uploads/attachment_data/ file/662499/hpr4217_mmr.pdf [Accessed 28 ]uly2018].
2 Moss W]. Measles. Lancet 2017:390:2490-502.

3 Centers for Disease Control and Prevention. Epidemiology and Prevention of Vaccine-Preventable Diseases. Measles, 2015;209-29. www.cdc.gov/vaccines/pubs/pinkbook/meas.html [Accessed 28 July2018]

4 Udupa S, Hameed T, Kovesi T. Pneumomediastinum and subcutaneous emphysema associated with pandemic (H1N1) influenza in three children. CMAJ 2011;183:220-2.

5 Mina MJ, Metcalf CJE, De Swart RL, Osterhaus ADME, Grenfell BT. Long-term measles-induced immunomodulation increases overall childhood infectious disease mortality. Science 2015;348:694-9.

Address for correspondence: Dr Azara Janmohamed, Imperial College Healthcare NHS Trust, Praed Street, London W2 1NY, UK

Email: a.janmohamed@nhs.net 